\title{
Matter-Antimatter Annihilation
}

\section{Ardeshir Irani}

The Dark Energy Research Institute, Downey, USA

Email: artirani@aol.com

How to cite this paper: Irani, A. (2021) Matter-Antimatter Annihilation. Journal of High Energy Physics, Gravitation and Cosmology, 7, 474-477.

https://doi.org/10.4236/jhepgc.2021.72027

Received: January 7, 2021

Accepted: April 3, 2021

Published: April 6, 2021

Copyright (c) 2021 by author(s) and Scientific Research Publishing Inc. This work is licensed under the Creative Commons Attribution International License (CC BY 4.0).

http://creativecommons.org/licenses/by/4.0/

\begin{abstract}
We know that when an electron, a matter particle, collides with a positron, an antimatter particle, they annihilate each other as the energy in the two particles is carried away by two real photons to conserve energy. The same phenomenon occurs as all matter annihilates an equal quantity of antimatter. In the case of charged particles like the proton and the antiproton, their opposite charges cancel while in the case of neutral particles like the neutron and the antineutron, their opposite spins cancel. In all cases, it is the cancellation of forward moving time for matter and backward moving time for antimatter that is responsible for the annihilation process having taken place. A reactor to produce energy for commercial use has been proposed based on matter antimatter collisions.
\end{abstract}

\section{Keywords}

Matter, Electron, Particle, Reactor

\section{The Cancellation of Time}

We consider the relationship between the Electric and Magnetic fields when viewed from two different reference frames. They are given by [1]:

$$
\begin{gathered}
E_{x}^{\prime}=E_{x} \\
E_{y}^{\prime}=c \gamma\left(E_{y} / c-\beta B_{z}\right) \\
E_{z}^{\prime}=c \gamma\left(E_{z} / c+\beta B_{y}\right) \\
B_{x}^{\prime}=B_{x} \\
B_{y}^{\prime}=\gamma\left(B_{y}+\beta E_{z} / c\right) \\
B_{z}^{\prime}=\gamma\left(B_{z}-\beta E_{y} / c\right)
\end{gathered}
$$

We pick the unprimed Electric and Magnetic fields as the rest frame for the charge $q$, and the primed Electric and Magnetic fields as the frame in which the 
charge $q$ is moving with velocity $\boldsymbol{v}$ along the $\mathbf{x}$-axis.

From Coulomb's Law in the rest frame:

$$
\bar{E}=q \times \hat{r} /\left(4 \pi \varepsilon_{0} r^{2}\right) \text { and } \bar{B}=0 .
$$

In spherical coordinates since:

$$
\hat{r}=\sin \theta \cos \phi \hat{x}+\sin \theta \sin \phi \hat{y}+\cos \theta \hat{z},
$$

we get:

$$
\begin{gathered}
E_{x}=q \sin \theta \cos \phi /\left(4 \pi \varepsilon_{0} r^{2}\right) \\
E_{y}=q \sin \theta \sin \phi /\left(4 \pi \varepsilon_{0} r^{2}\right) \\
E_{z}=q \cos \theta /\left(4 \pi \varepsilon_{0} r^{2}\right) \\
B_{x}=B_{y}=B_{z}=0
\end{gathered}
$$

Using the above relationships between primed and unprimed Electric and Magnetic fields, we get:

$$
\begin{gathered}
E_{x}^{\prime}=E_{x}=q \sin \theta \cos \phi /\left(4 \pi \varepsilon_{0} r^{2}\right) \\
E_{y}^{\prime}=\gamma E_{y}=\gamma q \sin \theta \sin \phi /\left(4 \pi \varepsilon_{0} r^{2}\right) \\
E_{z}^{\prime}=\gamma E_{z}=\gamma q \cos \theta /\left(4 \pi \varepsilon_{0} r^{2}\right) \\
B_{x}^{\prime}=0 \\
B_{y}^{\prime}=\gamma \beta E_{z} / c=\gamma \beta q \cos \theta /\left(4 \pi \varepsilon_{0} r^{2}\right) \\
B_{z}^{\prime}=-\gamma \beta E_{y} / c=-\gamma \beta q \sin \theta \sin \phi /\left(4 \pi \varepsilon_{0} r^{2}\right)
\end{gathered}
$$

The Energy Density $=$ Energy $/ \frac{4}{3} \pi r^{3}=\frac{1}{2} \varepsilon_{0}\left(E^{\prime}\right)^{2}+\left(B^{\prime}\right)^{2} / 2 \mu_{0}$

Hence

$$
\begin{aligned}
& \sqrt{\text { Energy }}=\sqrt{m c^{2}} \\
& = \pm q \sqrt{\frac{1}{24 \pi \varepsilon_{0} r}} \times\left\{\left(\sin ^{2} \theta \cos ^{2} \phi+\gamma^{2} \sin ^{2} \theta \sin ^{2} \phi+\gamma^{2} \cos ^{2} \theta\right)\right. \\
& \left.+\left(\gamma^{2}-1\right) \times\left(\cos ^{2} \theta+\sin ^{2} \theta \sin ^{2} \phi\right)\right\}^{1 / 2}
\end{aligned}
$$

If $\gamma=1$ the second term inside $\{()+()\}^{1 / 2}$ becomes 0 as it is due to the Magnetic field and the first term becomes 1 as it is due to the Electric field. We get the simpler [2] equation $\pm q \sqrt{\frac{1}{24 \pi \varepsilon_{0} r}}$ in this special situation as it is for the frame of reference in which the charge $q$ is at rest.

Energy can produce both positive and negative charges $+q$ and $-q$. Next let us examine what happens when a charged particle and antiparticle annihilate each other. If $+q$ and $-q$ both $\rightarrow 0$ that implies $m \rightarrow 0$ in accordance with the above equation, and that is exactly what happens when an electron and positron annihilate to produce two real photons of zero mass moving away with the same energy as the electron positron pair to conserve energy. 
The question then arises why positive and negative charges in a plasma do not annihilate one another. The reason is that besides opposite charges, opposite times must also cancel out. For the electron time moves in the forward direction while for the positron time moves in the backward direction. Hence time also cancels out leading to two photons which move at the speed of light for which time stands still.

The same rules must also apply for neutral matter and antimatter annihilation because neutral matter is made up of protons, electrons, and neutrons while neutral antimatter is made up of antiprotons, positrons, and antineutrons. While neutrons and antineutrons have no charge, they have opposite spin and hence their spins must cancel each other. The cancellation of spin should also apply to neutrinos and antineutrinos when they interact since they also carry opposite spin. CRT is a symmetry where C (charge conjugation) stands for matter or antimatter charge, $\mathrm{R}$ stands for rotational spin clockwise or counterclockwise, and $\mathrm{T}$ stands for time moving forward or backward. Hence, we need cancellation of charge for charged particles and antiparticles, cancellation of spin for neutral particles and antiparticles, but cancellation of time in all cases which is the condition that must always be met for particle-antiparticle annihilation to take place.

\section{Particle Antiparticle Reactors}

Antimatter has been considered as a trigger mechanism for nuclear weapons, and as the possibility of creating thermonuclear energy [3]. We propose an alternative method of creating energy by shooting electron-positron or proton-antiproton beams at each other and using the energy released as a means of producing commercial energy as in a fission reactor without the radioactive byproducts. This type of energy production should be compared to the proposed fusion reactors such as magnetically confined or inertially confined fusion with the advantage of $100 \%$ conversion of the mass in the two beams into pure energy, a conversion factor that neither fission nor fusion can reproduce, and without the need for raw materials uranium-235 or plutonium-239 that would be needed for fission, or deuterium and tritium needed for fusion reactions to take place.

\section{Conclusion}

A reactor in which antiproton beams are made to collide with electron beams of the same velocity should be an advantageous scenario to consider since the antiproton is 1836 times heavier than the electron. Hence for the same beam volumes (equal length and radius), the ratio of the densities of the antiproton beam to the electron beam would be $5.45 \times 10^{-4}$, thereby reducing the necessity of producing intense antiproton beams.

\section{Conflicts of Interest}

The author declares no conflicts of interest regarding the publication of this paper. 


\section{References}

[1] Fleisch, D. (2019) A Student's Guide to Vectors and Tensors. 182.

[2] Irani, A. (2021) Dark Energy, Dark Matter, and the Multiverse. Journal of High Energy Physics, Gravitation and Cosmology, 7, 172-190.

https://doi.org/10.4236/jhepgc.2021.71009

[3] Gsponer, A. and Jean-Pierre, H. (1987) The Physics of Antimatter Induced Fusion and Thermonuclear Explosions. In: Velarde, G. and Minguez, E. (Eds.), Proceedings of the $4^{\text {th }}$ International Conference on Emerging Nuclear Energy Systems, Madrid, 30June-4 July 1986, 166-169. arXiv:physics/0507114 